\title{
The Effects of Maternal Omega-3 Fatty Acid Supplementation on Breast Milk Fatty Acid Composition
}

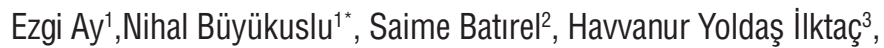 \\ Muazzez Garipağaoğlu \\ 'Department of Nutrition and Dietetics, School of Health Sciences, Istanbul Medipol University, Istanbul, Turkey. \\ ${ }^{2}$ Department of Medical Biochemistry, Faculty of Medicine, Marmara University, Istanbul, Turkey. \\ ${ }^{3}$ Department of Nutrition and Dietetics, School of Health Sciences, Istanbul Medeniyet University, Istanbul, Turkey. \\ ${ }^{4}$ Department of Nutrition and Dietetics, School of Health Sciences, Fenerbahçe University, Istanbul, Turkey.
}

\begin{abstract}
Breast milk is the first source of omega-3 fatty acids (FA) for infants. We hypothesized that maternal omega-3 FAs supplementation affects the FA composition of breast milk. Thirtysix women received $950 \mathrm{mg}$ omega-3 polyunsaturated (PUFA) supplementation per day for 9 months from 22-24 weeks of pregnancy until the 6 months of lactation and 26 women were enrolled as controls. Demographic data and nutritional status were taken by a questionnaire. Breast milk samples were collected at just after birth and 6 months of lactation. Fatty acids were analyzed by gas chromatography mass spectrometry (GC-MS). Data was assessed using SPSS 22.0 software. The supplementation increased the level of docosahexaenoic acid (DHA) and eicosapentaenoic acid (EPA) while decreased the level of saturated fatty acids (SFA) in breast milk. The ratio of omega-6/omega-3 FAs was also decreased in the supplemented mothers' milk. In conclusion the maternal supplementation of omega-3 FAs improved the DHA and EPA levels.
\end{abstract}

Keywords: Docosahexaenoic acid (DHA); eicosapentaenoic acid (EPA); omega-3 fatty acids; omega supplement; breast milk.

\section{INTRODUCTION}

The lipid content of mature breast milk is 3.2-4.8\%. ${ }^{1}$ This amount of lipid provides approximately $40-50 \%$ of daily energy demand for infants. Nutritional status of mother is one of the major factors that affects the milk content. ${ }^{2}$ There is a correlation between carbohydrate intake of mothers and fat content in breast milk. ${ }^{3}$ Diet rich in carbohydrates results in higher levels of saturated fatty acids. ${ }^{4}$ In addition, there are several studies showed the association between mother's diet and breast milk fat content..$^{5-8}$

*Corresponding author: Nihal Büyükuslu, e-mail: nbuyukuslu@medipol.edu.tr (Received 22 January 2018, accepted 03 March 2018) 
Human breast milk has the ideal composition for feeding infants to meet all necessary nutrients for growth and development. The fatty acid composition of breast milk varied in the mothers from different countries all around the world. For instance, milk samples from Spanish mothers contain high monounsaturated fatty acids (MUFAs) (39.63 $\pm 3.57 \%) .{ }^{9}$ On the other hand, milk samples from Nigerian mothers contain high saturated fatty acids (SFAs) (54.07\%). ${ }^{10}$ In the mothers from Kenya the percentage of the SFAs ( $16 \%)$ was significantly lower than the percent of the unsaturated fatty acids in breast milk. ${ }^{11}$

Long chain polyunsaturated fatty acids (LC-PUFAs) contain two principal families: omega-6 PUFAs including linoleic acid (LA, 18:2n6), arachidonic acid (AA, 22:4n6), and omega-3 PUFAs including alpha linolenic acid (ALA, 18:3n3), eicosapentaenoic acid (EPA, 20:5n3), docosapentaenoic acid (DPA, 22:5n3) and docosahexaenoic acid (DHA, 22:6n3). LC-PUFAs are essential for normal neonatal mammalian development. Breast milk is the unique source of the LCPUFAs for breastfed infants. The levels of DHA, EPA and AA are elevated in fetus brain and associated with the development of the central nervous and visual sytems. ${ }^{12,13}$ The level of DHA in breast milk varies between $0.06 \%$ to $1.4 \%$ of total fatty acids with a mean of $0.32 \pm 0.22 \%$ worldwide average. ${ }^{14}$ In a comparative study, it was shown that the DHA levels were $0.67 \pm 0.32 \%$ in Asia (excluding China), $0.37 \pm 0.15 \%$ in China, $0.34 \pm 0.14 \%$ in Europe, $0.32 \pm 0.30 \%$ in Africa and the lowest in America (0.20 $\pm 0.11 \%) .{ }^{15}$ The amount of DHA in the breast milk of the women who had high fish intake was higher than the ones who consumed less fish. The DHA levels in the milk of women from Surinam, St Lucia, Malaysia, Dominica and Curaco were found higher than the western style diet such as in USA and many European countries. ${ }^{16-20}$

Since omega- 3 FAs can only be obtained from the diet, the omega- 3 rich foods and supplements should be adviced to women during pregnancy and lactation. The major sources of omega-3 FAs are fish, flaxseed, canola and soybean oils. However, overconsumption of certain fish may cause high mercury intake and can cause toxicity. ${ }^{21}$ Therefore omega- 3 FAs from vegetable sources and the supplements containing DHA and EPA are recommended to the mothers. ${ }^{22,23}$ The daily recommended DHA intake is an average of $\geq 200 \mathrm{mg} /$ day in pregnancy and lactation. ${ }^{24}$ We previously studied the impact of DHA+EPA supplementation during the last trimester of pregnancy and found an increase in cord blood. ${ }^{25}$ We extended the study to investigate the further effect of omega-3 supplement in lactation for 6 months and hypothesized that maternal supplementation would enhance breast milk fatty acid composition. To our knowledge this is the first study to cover both pregnancy and lactation for a long term (nine months) omega-3 FA supplementation. 


\section{METHODOLOGY}

\section{Study population and design}

This experimental study was conducted on 62 healthy, singleton voluntary pregnant women between September 2015 and December 2016 in Istanbul, Turkey. The mothers were informed about the study at 22-24 weeks of pregnancy during routine prenatal care visits in the hospital. Among the interviewees thirtysix pregnants accepted to take the supplement daily during the last trimester of pregnancy and the 6 month of lactation period and this group was named as omega-3 group. Twenty-six women participitated in the study without taking supplementation and this group was named as control group. They were asked to sign an informed consent form and to answer the questions in a survey including the demographic and anthropometric data, daily energy and fat consumption. Breast milk samples were taken twice: just after birth and 6 months of lactation. The pregnant women who gave preterm birth and had a history of chronic diseases and/or consumed omega-3 fatty acid supplements were excluded. Six to $10 \mathrm{~mL}$ of breast milk samples were collected from each subject in the morning between 9.00 and 11.00 am. The samples immediately were carried to the laboratory in an ice-cold package, filled in vials and stored at $-80 \mathrm{C}^{\circ}$ until analysis.

All procedures and protocols received prior approval by the Istanbul Medipol University Ethics and Research Committee.

\section{Suplementation}

The participants were assigned to receive a softgel capsule/day as the supplement (Martek Biosciences Corporation, Solgar, Leonia, NJ, USA), starting from last trimester to the end of 6th month of lactation. One capsule provides 378 mg docosahexaenoic acid (DHA) and $504 \mathrm{mg}$ eicosapentaenoic acid (EPA) in total 950 mg omega-3 PUFA. Control group had no supplement or placebo. No dietary instructions were given to both groups.

\section{Analysis of fatty acids}

The milk samples were thawed and lipids were extracted according to Bligh and Dyer method. ${ }^{26}$ Fatty acid composition of extracted lipids was determined by conversion into fatty acid methyl esters (FAME). Trans-methylation was performed in methanolic- $\mathrm{HCl}(0.5 \mathrm{~N})$ at $80^{\circ} \mathrm{C}$. The FAME were separated and analyzed by gas chromatography - mass spectrometry (GC-MS) (Shimadzu QP-2010, Kyoto, Japan). Thirty meters of fused-silica capiller colomn was used. Standardization was performed using a standart mixture involving 37 fatty acids. Their retention times were recorded and fatty acid library was maintained. Chromatograms were analysed in terms of \% by weight of total fatty acids. 


\section{Statistical analyses}

Statistical Package for Social Sciences version 22.o (SPSS, Chicago, IL, USA) was used for statistical analyses. The results were presented as mean $\pm \mathrm{SD}$. The Student's $t$ test was applied for paired and independent values and their nonparametric equivalent to evaluate significant differences. Pearson coefficient of correlation was used to analyze relationships between numerical variables. Statistically significancy accepted as the level of $\mathrm{p}<0.05$. Considering a 0.05 two-sided significance level and large effect size (o.80), the power was 0.80 .

\section{RESULTS}

\section{The baseline characteristics and nutritional status of the mothers}

The demographic characteristics of mothers were shown in Table 1 . The mean ages of women in omega-3 group and control group were $31.8 \pm 4.3$ years and $30.4 \pm 3.9$ years respectively. BMI values of women at pre-pregnancy were $22.9 \pm 3.1 \mathrm{~kg} / \mathrm{m}^{2}$ (omega-3 group, $\mathrm{n}=36$ ) and $23.0 \pm 2.5 \mathrm{~kg} / \mathrm{m}^{2}$ (control group, $\mathrm{n}=26$ ). There was no significancy for each parameter indicating the homogeneity of the pregnants participitated in the study.

Table 1. Demographic characteristics of women*

\begin{tabular}{|l|c|c|c|}
\hline & Control group & Omega-3 group & $P$ \\
\hline N & 26 & 36 & \\
\hline Age (year) & $30.4 \pm 3.9$ & $31.8 \pm 4.3$ & 0.168 \\
\hline Pre-pregnancy BMI $\left(\mathrm{kg} / \mathrm{m}^{2}\right)$ & $23.0 \pm 2.5$ & $22.9 \pm 3.1$ & 0.550 \\
\hline
\end{tabular}

*Data recorded at 22-24 weeks of pregnancy

During lactation period, the daily energy and fat intake was recorded once at the $3^{\text {rd }}$ month and results were shown in Table 2. The comparison of two groups revealed no significance in the daily intake of energy, carbohydrate, protein and fat. There were no significant differences in daily MUFA and PUFA intakes between two groups $(\mathrm{p}<0.05)$ but the daily SFA intake was found significantly different $(\mathrm{p}>0.05)$. 
Table 2. Daily energy and fat intakes at the $3^{\text {rd }}$ month of lactation

\begin{tabular}{|c|c|c|c|}
\hline & Control group & Omega-3 group & $P$ \\
\hline Energy $(\mathrm{kcal})$ & $1789.0 \pm 439.1$ & $1804.7 \pm 614.7$ & 0.865 \\
\hline Fat $(\mathrm{g})$ & $80.1 \pm 24.7$ & $86.0 \pm 33.1$ & 0.594 \\
\hline Fat $(\%)$ & $41.3 \pm 7.7$ & $42.6 \pm 5.7$ & 0.515 \\
\hline SFA $(\mathrm{g})$ & $27.5 \pm 7.4$ & $33.5 \pm 12.4$ & $0.038^{*}$ \\
\hline MUFA (g) & $30.0 \pm 11$ & $32.7 \pm 13.9$ & 0.541 \\
\hline PUFA $(\mathrm{g})$ & $16.2 \pm 9.3$ & $13.6 \pm 9.1$ & 0.234 \\
\hline
\end{tabular}

"Statistical significance at $\mathrm{p}<0.05$ level

\section{Fatty acid distribution in breast milk}

The effect of omega-3 supplementation during pregnancy and lactation on FA composition of breast milk was demonstrated in Table 3. The omega-3 FA supplementation decreased total SFA in colostrum and mature milk. However, the only significant difference was in mature milk ( $\mathrm{p}<0.05)$. Total MUFAs was higher in colostrum but slightly lower in mature milk at 6 months with no significancy in omega-3 supplemented group. Total PUFAs were increased in both milk sampling occasions. The only significant difference was in colostrum $(\mathrm{p}<0.05)$.

Table 3. Fatty acid composition of mothers' milk

\begin{tabular}{|c|c|c|c|c|c|c|}
\hline & \multicolumn{3}{|c|}{ Colostrum } & \multicolumn{3}{c|}{ Mature milk (6 months) } \\
\cline { 2 - 7 } & $\begin{array}{c}\text { Control } \\
\text { group } \\
(\mathrm{n}=26)\end{array}$ & $\begin{array}{c}\text { Omega-3 } \\
\text { group } \\
(\mathrm{n}=28)\end{array}$ & $P$ & $\begin{array}{c}\text { Control } \\
\text { group } \\
(\mathrm{n}=18)\end{array}$ & $\begin{array}{c}\text { Omega-3 } \\
\text { group } \\
(\mathrm{n}=26)\end{array}$ & $\mathrm{P}$ \\
\hline Total SFA & $44.84 \pm 3.93$ & $43.15 \pm 6.27$ & 0.059 & $44.42 \pm 4.72$ & $41.50 \pm 6.25$ & $0.038^{\star}$ \\
\hline Total MUFA & $35.21 \pm 3.40$ & $36.52 \pm 6.43$ & 0.144 & $35.34 \pm 1.71$ & $35.25 \pm 4.16$ & 0.148 \\
\hline Total PUFA & $19.08 \pm 3.56$ & $21.35 \pm 4.66$ & $0.047^{\star}$ & $20.20 \pm 4.66$ & $22.46 \pm 5.41$ & 0.080 \\
\hline Total omega-3 & $1.32 \pm 0.32$ & $1.58 \pm 0.38$ & 0.083 & $1.26 \pm 0.31$ & $1.65 \pm 0.71$ & 0.142 \\
\hline DHA 22:6n3 & $0.36 \pm 0.10$ & $0.51 \pm 0.18$ & $0.000^{* *}$ & $0.22 \pm 0.10$ & $0.31 \pm 0.23$ & $0.000^{\star *}$ \\
\hline EPA 20:5n3 & $0.09 \pm 0.04$ & $0.16 \pm 0.07$ & $0.000^{* *}$ & $0.08 \pm 0.03$ & $0.14 \pm 0.08$ & 0.232 \\
\hline Total omega-6 & $17.80 \pm 3.59$ & $19.77 \pm 4.52$ & $0.034^{*}$ & $18.93 \pm 4.69$ & $20.81 \pm 5.26$ & 0.094 \\
\hline $\begin{array}{c}\text { Omega-6/ } \\
\text { Omega-3 }\end{array}$ & $14.31 \pm 4.72$ & $12.97 \pm 3.62$ & 0.723 & $16.32 \pm 8.13$ & $13.79 \pm 4.16$ & 0.872 \\
\hline
\end{tabular}

The fatty acids were given $\%$ by weight of total fats

${ }^{\star}$ Statistical significance at $p<0.05$ level; ${ }^{*}$ Statistical significance at $p<0.000$ level 
Total omega-3 FAs levels were higher in colostrum and $6^{\text {th }}$ month milk. Among omega-3 FAs the levels of DHA and EPA were significantly increased in colostrum. Both DHA and EPA levels in mature milk at 6 months were higher in supplemented groups with the only significance at DHA level. The levels of total omega-6 FAs were higher in colostrum $(\mathrm{p}<0.05)$ and mature milk $(\mathrm{p}>0.05)$ samples from supplemented mothers.

The ratio of omega- 6 FAs to omega-3 FAs was lower in colostrum and at 6 months from supplemented mothers. The omega-6/omega-3 ratios in both colostrum and $6^{\text {th }}$ month samples were lower in the supplemented mother milks. None of the differences were significant.

\section{The levels of DHA and EPA in breast milk}

Omega-3 supplementation increased the levels of DHA and EPA in both colostrum and mature milk. The differences between control and supplemented groups were significant for DHA levels for colostrum ( $\mathrm{p}<0.001)$ and mature milk $(\mathrm{p}<0.001)$ but EPA levels were significant in colostrum $(\mathrm{p}<0.001)$ but not in mature milk ( $>0.05$ ). The total omega-6 FAs of supplemented mothers were higher in colostrum $(\mathrm{p}<0.05)$ and mature milk $(\mathrm{p}>0.05)$ than the control mothers had. The rate of omega- 6 to omega- 3 was affected by the omega-3 FAs supplementation. The ratios decreased in colostrum (from $14.31 \pm 4.72$ to $12.97 \pm 3.62$ ) and at $6^{\text {th }}$ months milk (from $16.32 \pm 8.13$ to $13.79 \pm 4.16$ ) depending on the increased amounts of omega- 3 FAs indicating the beneficial influence of maternal omega-3 supplementation on omega-3 FAs quality of mother milk.

\section{DISCUSSION}

This study was designed to compare the levels of fatty acids in breast milk samples between control and omega supplemented groups during the last trimester of their pregnancy and 6 months of lactation period. Within our knowledge this is the longest omega-3 supplementation for mothers during lactation. ${ }^{27}$ There are several studies indicate that the composition of fatty acids in breast milk changes through the lactation period. The content of human milk is affected by nutritional habits, cultural and social differences, maternal and environmental factors. ${ }^{1}$ A study on the prediction of cognitive test performance associated with the fatty acid content included the level of fatty acids in different countries. In mentioned study, the mean levels of fatty acids were $43.32 \pm 9.56 \%$ SFAs (33 countries), $35.69 \pm 6.45 \%$ MUFAs (34 countries), $14.53 \pm 3.82 \%$ PUFAs (33 countries) and $0.73 \pm 0.38 \%$ LC omega-3 FAs (30 countries). The concentrations of omega- 3 fatty acids were reported as $0.38 \pm 0.23 \%$ DHA (50 countries), $0.19 \pm 0.09 \%$ DPA (33 countries) and $0.13 \pm 0.09 \%$ EPA (36 countries). ${ }^{28}$ As the content of breast milk is highly related to mother's diet, daily intake of DHA-rich 
foods as much as foods containing industrial trans fatty acid, supplements, processed foods and meats would be the major indicators of the distribution of fatty acids in milk. ${ }^{29}$ The FAs composition of mature breast milk of Israeli women was analysed and it was found that the contents of total SFAs, total MUFAs and total PUFAs were $42 \pm 7 \%, 33 \pm 5 \%$ and $24 \pm 4 \%$ of total FAs respectively. ${ }^{30}$ In a study on Turkish women, Aydın et al. showed higher level of SFA in total FAs of breast milk ( $46.57 \%$ at 7 days, $51.56 \%$ at 28 days of lactation) than predicted in the control group of current study $(44.84 \pm 3.93 \%$ in colostrum and $44.42 \pm 4.72 \%$ at 15 days of lactation). ${ }^{31}$ Omega-3 supplementation further reduced the values. The fluctuations between the fatty acid levels in different countries might arise from the cultural habits, regional and seasonal differences in nutritional behaviours. In a study by Lopez-Lopez the omega-6/omega-3 FAs ratios in breast milk of Spanish women were very similar the ratios in our study. ${ }^{9}$ This similarity may reflect the Meditterranean style nutrition in both Spain and Turkey.

Linoleic acid and ALA are the precursors of LC-PUFAs. The conversion of these precursors into AA, EPA and DHA is low. ${ }^{32,33}$ As the beneficial effects of omega-3 fatty acids for infant development are inevitable, any touch to increase omega-3 FAs level in breast milk is appreciable. The reported average concentration of DHA in human milk Worldwide is $0.32 \pm 0.22 \%$. In the current study, Turkish women had the level of DHA min. $0.22 \pm 0.10 \%$ ( $6^{\text {th }}$ month) and max. $0.36 \pm 0.10$ (colostrum). After DHA+EPA supplementation during pregnancy and lactation the levels increased to $0.31 \pm 0.23$ ( $6^{\text {th }}$ month) and $0.51 \pm 0.18$ (colostrum).

There are several studies which demonstrate that omega- 3 supplementation to mothers during lactation may influence its concentration in human breast milk. Bortoloza et al measured the DHA and EPA levels after a daily supplementation with fish oil capsules that corresponded to a daily intake of $315 \mathrm{mg}$ of DHA and $80 \mathrm{mg}$ of EPA during the third trimester of pregnancy and the first three months postpartum. They found that the milk of women taking fish oil had higher DHA levels after delivery (0.324\% at $30^{\text {th }}$ day, $0.207 \%$ at $90^{\text {th }}$ day) comparing with the DHA levels of control mothers' milk (0.104\% at $30^{\text {th }}$ day, $0.030 \%$ at $90^{\text {th }}$ day). The EPA levels at 30 and 90 days were $0.109 \%$ and $0.107 \%$ in omega group and $0.084 \%$ and $0.050 \%$ in control group in the same study. ${ }^{34} \mathrm{Smit}$ et al investigated that DHA status of malnourished children was strongly dependent on the omega-3 fatty acid intake from breast milk. ${ }^{35}$ In the present study, we also found an increase of DHA and EPA levels in colostrum and in mature milk samples from the supplemented mothers. In colostrum, the DHA and EPA differences between the groups were statistically significant at the level of $p=0.000$ indicating the improving effect of the omega fatty acid supplementation on the amount of DHA and EPA in human milk. In addition, it was observed that supplementation had 
more influence on colostrum than mature milk at 6 months. The rise in omega- 3 fatty acids is beneficial in case of the aid in development of infant. Similarly, Jensen et al determined that DHA supplementation (170-260 mg/day) of breastfeeding mothers increased the DHA contents of breast milk. They also found the strong positive correlations between maternal plasma-phospholipid DHA and EPA and the contents of these fatty acids in breast milk supporting the idea that of supplementation of lactating women with DHA might be the most reliable means of increasing breast-milk DHA ${ }^{36}$ The effects of DHA supplementation on the fatty acid composition of breast milk in lactating women were investigated in a study by Sherry et al. They found that saturated fatty acids were the most abundant in breast milk at baseline. After low (200 mg/day) and high dose (400 mg/day) of DHA supplementation for 6 weeks, the level of DHA in breast milk was significantly higher compared with placebo. The levels of DHA were elevated from $6.98 \pm 0.94 \%$ to $8.83 \pm 1.03 \%$ for low dose and from $5.14 \pm 0.51 \%$ to $13.08 \pm 1.69 \%$ for high dose supplementation. ${ }^{37}$ The results support our findings that DHA suplementation in lactation period increase the DHA level and decrease SFAs in breast milk. Moreover, Much et al reported that the DHA levels at $6^{\text {th }}$ and $16^{\text {th }}$ weeks postpartum were $0.28 \pm 0.14 \%$ and $0.24 \pm 0.13 \%$ respectively. After diet intervention, they increased to $1.34 \pm 0.67 \%$ and $1.12 \pm 0.39 \%$ pointing out the efficieny of DHA supplementation spesifically at early times of lactation in a similarity to our results..$^{8}$

In our daily life, the consumption of n-6 fatty acids are increased compared to the traditional diet due to widely used industrially processed fat. ${ }^{39}$ We defined that the ratios of omega-6/omega-3 were between 10.7 and 14.2 for omega supplemented mothers and between 11.9 and 15.0 for control mothers. That means that the supplementation of omega-3 FAs lowers the ratio of omega- 6 to omega-3 FAs in the breast milk in comparison with control mothers.

This study has some limitations. The numbers of mothers for each group were not equal because of elimination of mothers for several reasons such as being away from city, diseases of mothers or babies at one or more stages of the study. The questionnaire used for evaluation of demographic and nutritional status was based on self-report. The nutritional status was reported once at $3^{\text {rd }}$ month of lactation. Omega-3 supplementation was voluntarily performed. Although there were a good correlation betwen the numbers of the supplement packages and the days that the mothers took the supplements, nine months period was quite long time for mothers to follow daily requirements for supplement. However, all participants verbally declared that they took one capsule omega in a day. 
In conclusion, the supplementation of omega-3 FAs for nine months starting from the beginning of the last trimester to the $6^{\text {th }}$ month of lactation improves the DHA and EPA levels significantly in colostrum and the mature milk and decreases the ratio of omega-6/omega-3 FAs and saturated fatty acid concentrations of breast milk. Further studies need to be proceeded on the factors affecting FAs composition of breast milk for omega-3 supplemented mothers and optimize the concentration of omega-3 FAs intaken during lactation for better development of infants.

\section{CONFLICT OF INTEREST}

The authors declare no conflict of interest.

\section{REFERENCES}

1. Ballard, O.; Morrow, A.L. Human milk composition: Nutrients and bioactive factors. Pediatr. Clin. North. Am. 2013, 6o, 49-74.

2. Martin, M.A.; Lassek, W.D.; Gaulin, S.J.C.; Evans, R.W.; Woo, J.G.; Geraghty, S.R.; et al., Fatty acid composition in the mature milk of Bolivian forager-horticulturalists: Controlled comparisons with a US sample. Matern. Child Nutr. 2012, 8, 404-418.

3. Nikniaz, L.; Mahdavi, R.; Arefhoesseini, S.R.; Khiabani, M. S. Association between fat content of breast milk and maternal nutritional status and infants' weight in Tabriz. Iran. Malays. $J$. Nutr. 2009, 15, 37-44.

4. Delplanque, B.; Gibson, R.; Koletzko, B.; Lapillonne, A.; Strandvik, B. Lipid quality in infant nutrition: Current knowledge and future opportunities. J. Pediatr. Gastroenterol. Nutr. Discipline. 2015, 61, 8-17.

5. Kelishadi, R.; Hadi, B.; Iranpour, R.; Khosravi-Darani, K.; Mirmoghtadaee, P.; Farajian S. et al., A study on lipid content and fatty acid of breast milk and its association with mother's diet composition. J. Res. Med. Sci. 2012, 9, 824-827.

6. Villalpando, S.; del Prado, M. Interrelation among dietary energy and fat intake, maternal body fatness and milk total lipids in humans. J. Mammary Gland. Biol. Neoplasia. 1999, 4, 285-295.

7. Peng, Y.; Zhou, T.; Wang, Q.; Liu, P.; Zhang, T.; Zetterström, R. et al., Fatty acid composition of diet, cord blood and breast milk in Chinese mothers with different dietary habits. PLEFA, 2009, 81, 325-330.

8. Michaelsen, K.F.; Larsen, P.S.; Thomsen, B.L.; Samuelson, G. The Copenhagen cohort study on infant nutrition and growth: Breast-milk intake, human milk macronutrient content, and influencing factors. Am. J. Clin. Nutr. 1994, 59, 600-611.

9. Lopez-Lopez, A.; Lopez-Sabater, M.C.; Campoy-Folgoso, C.; Rivero-Urgell, M.; CastelloteBargallo, A.I. Fatty acid and $s n-2$ fatty acid composition in human milk from Granada (Spain) and in infant formulas. Eur. J. Clin. Nutr. 2002, 56, 1242-1254.

10. Koletzko, B.; Thiel, I.; Abiodun, P.O. Fatty acid composition of mature human milk in Nigeria. Zernahrungwiss. 1991, 30, 289-297. 
11. Kiprop, J.V.; Girard, A.W.; Gogo, L.A.; Omwamba, M.N.; Mahungu, S. M. Determination of the fatty acid profile of breast milk from nursing mothers in Bungoma County, Kenya. Food Nutr. Sci. 2016, 7, 661-670.

12. Bernardi, J.R.; Escobar, R.S.; Ferreira, C.F.; Silveira, P.P. Fetal and neonatal levels of omega-3: Effects on neurodevelopment, nutrition, and growth. Scientific World Journal. 2012, 2012: 202473 .

13. Lauritzen, L.; Brambilla, P.; Mazzocchi, A.; Harslof, L.B.; Ciappolino, V.; Agostoni, C. DHA effects in brain development and function. Nutrients 2016, 8, 6. doi:10.3390/nu8010006.

14. Brenna, J.T.; Varamini, B.; Jensen, R.G.; Diersen-Schade, D.A.; Boettcher, J.A.; Arterburn, L.M. Docosahexaenoic and arachidonic acid concentrations in human breast milk worldwide. Am. J. Clin. Nutr. 2007, 85, 1457-1464.

15. Li, S.Y.; Dong, X.L.; Wong, W.S.V.; Su, Y.X.; Wong, M.S. Long-chain polyunsaturated fatty acid concentrations in breast milk from Chinese mothers: Comparison with other regions. Int. J. Child Health Nutr. 2015, 4, 230-239.

16. Muskiet, F.A.; Hutter, N.H.; Martini, I.A.; Jonxis, J.H.; Offringa, P.J.; Boersma, E.R. Comparison of the fatty acid composition of human milk from mothers in Tanzania, Curacao and Surinam. Hum. Nutr. Clin. Nutr. 1987, 41, 149-159.

17. Boersma, E.R.; Offringa, P.J.; Muskiet, F.A.; Chase, W.M.; Simmons, I.J. Vitamin E, lipid fractions, and fatty acid composition of colostrum, transitional milk, and mature milk: an international comparative study. Am. J. Clin. Nutr. 1991, 53, 1197-1204.

18. Kneebone, G.M.; Kneebone, R.; Gibson, R.A. Fatty acid composition of breast milk from three racial groups from Penang, Malaysia. Am. J. Clin. Nutr. 1985, 41, 765-769.

19. Koletzko, B.; Thiel, I.; Abiodun, P.O. The fatty acid composition of human milk in Europe and Africa. J. Pediatr. 1992, 120, S62-70.

20. Salamon, Sz.; Csapo, J. Composition of the mother's milk II. Fat contents, fatty acid composition. A review. Acta Univ. Sapientiae, Alimentaria. 2009, 2, 196-234.

21. WHO, Preventing disease through healthy environments. Exposure to mercury: A major public health concern. 2007. Geneva, World Health Organization.

22. Coletta, J.M.; Bell, S.J.; Roman, A.S. Omega-3 fatty acids and pregnancy. Rev. Obstet. Gynecol. 2010, 3, 163-171.

23. Greenberg, J.A.; Bell, S.J.; Van Ausdal, W. Omega-3 fatty acid supplementation during pregnancy. Rev. Obstet. Gynecol. 2oo8, 1, 162-169.

24. FAO, Fats and fatty acids in human nutrition. Report of an expert consultation. FAO Food Nutr. Pap. 2008, 91, 63-76.

25. Büyükuslu, N.; Ovalı, S.; Altuntaş, Ş.L.; Batırel, S.; Yiğit, P.; Garipağaoğlu, M. Supplementation of docosahexaenoic acid (DHA) / Eicosapentaenoic acid (EPA) in a ratio of 1 / 1.3 during last trimester of pregnancy results in EPA accumulation in cord blood. PLEFA. 2017, 125(1), 32-36.

26. Bligh, E.G.; Dyer, W.J. A rapid method of total lipid extraction and purification. Can. J. Biochem. Physiol. 1959, 37, 911-917.

27. Li G, Chen H, Zhang W, Tong Q, Yan Y. Effects of maternal omega-3 fatty acids supplementation during pregnancy/lactation on body composition of the offspring: A systematic review and meta-analysis. Clin.Nutr. 2017, 1-12. 
28. Lassek, W.D.; Gaulin, S.J.C. Linoleic and docosahexaenoic acids in human milk have opposite relationships with cognitive test performance in a sample of 28 countries. PLEFA. 2014, 91, 195-201.

29. Bradbury, J. Docosahexaenoic Acid (DHA): An ancient nutrient for the modern human brain. Nutrients. 2011, 3, 529-554.

30. Saphier, O.; Blumenfeld, J.; Silberstein, T.; Tzor, T.; Burg, A. Fatty acid composition of breastmilk of Israeli mothers. Indian Pediatr. 2013, 50, 1044-1046.

31. Aydın, I.; Turan, O.; Aydın, F.N.; Koç, E.; Hirfanoğlu, I.M.; Akyol, M. et al., Comparing the fatty acid levels of preterm and term breast milk in Turkish women. Turk J. Med. Sci. 2014, 44, 305-310.

32. Burdge, G.C.; Jones, A.E.; Wootton, S.A. Eicosapentaenoic and docosapentaenoic acids are the principal products of a-linolenic acid metabolism in young men. Br. J. Nutr. 2002, 88, 355-364.

33. Burdge, G.C.; Wootton, S.A. Conversion of $\alpha$-linolenic acid to eicosapentaenoic, docosapentaenoic and docosahexaenoic acids in young women. Br. J. Nutr. 2002, 88, 411-420.

34. Bortolozo, E.A.F.Q.; E. Sauer, Supplementation with the omega-3 docosahexaenoic acid: Influence on the lipid composition and fatty acid profile of human milk. Rev. Nutr. Campinas. 2013, 26, 27-36.

35. Smit, E.N.; Oelen, E.A.; Seerat, E.; Muskiet, F.A.J.; Boersma, E.R. Breast milk docosahexaenoic acid (DHA) correlates with DHA status of malnourished infants. Arch. Dis. Child. 2ooo, 82, 493-494.

36. Jensen, C.L.; Maude, M.; Anderson, R.E.; Heird, W.C. Effect of docosahexaenoic acid supplementation of lactating women on the fatty acid composition of breast milk lipids and maternal and infant plasma phospholipids. Am. J. Clin. Nutr. 2000, 71, 292S-299S.

37. Sherry, C.L.; Oliver, J.S.; Marriage, B.J. Docosahexaenoic acid supplementation in lactating women increases breast milk and plasma docosahexaenoic acid concentrations and alters infant omega 6:3 fatty acid ratio. PLEFA. 2015, 95, 63-69.

38. Much, D.; Brunner, S.; Vollhardt, C.; Schmid, D.; Sedlmeier, E.M.; Bruder, M. et al. Breast milk fatty acid profile in relation to infant growth and body composition: results from the INFAT study. Pediatr. Res. 2013, 74, 230-237.

39. Simopoulos, A. P. An increase in the omega-6/omega-3 fatty acid ratio increases the risk for obesity. Nutrients. 2016, 8, 128-145. 
